MIMR-DGSA: Unsupervised Hyperspectral Band Selection Based on Information Theory and a Modified Discrete Gravitational Search Algorithm

Julius Tschannerl, Jinchang Ren, Peter Yuen, Genyun Sun, Huimin Zhao, Zhijing Yang, Zheng Wang, Stephen Marshall

PII: S1566-2535(18)30066-6

DOI: https://doi.org/10.1016/j.inffus.2019.02.005

Reference: INFFUS 1079

To appear in: Information Fusion

Received date:

25 January 2018

Revised date:

12 February 2019

Accepted date:

14 February 2019

Please cite this article as: Julius Tschannerl, Jinchang Ren, Peter Yuen, Genyun Sun, Huimin Zhao, Zhijing Yang, Zheng Wang, Stephen Marshall, MIMR-DGSA: Unsupervised Hyperspectral Band Selection Based on Information Theory and a Modified Discrete Gravitational Search Algorithm, Information Fusion (2019), doi: https://doi.org/10.1016/j.inffus.2019.02.005

This is a PDF file of an unedited manuscript that has been accepted for publication. As a service to our customers we are providing this early version of the manuscript. The manuscript will undergo copyediting, typesetting, and review of the resulting proof before it is published in its final form. Please note that during the production process errors may be discovered which could affect the content, and all legal disclaimers that apply to the journal pertain. 


\section{Highlights}

- VarBWFastMI allows fast calculation of pairwise mutual information of HSI datasets

- Mutual information and entropy are used to evaluate the MIMR criterion

- A new DGSA with fewer parameters is used to generate band subsets

- Spatial-spectral fusion based neighbourhood concept for band subsets is established 
Information Fusion, Volume 51, November 2019, pp. 189-200

DOI: $10.1016 /$ j.inffus.2019.02.005

\title{
MIMR-DGSA: Unsupervised Hyperspectral Band Selection Based on Information Theory and a Modified Discrete Gravitational Search Algorithm
}

\author{
Julius Tschannerl ${ }^{\mathrm{a}}$, Jinchang Ren ${ }^{\mathrm{a}}$, Peter Yuen ${ }^{\mathrm{b}}$, Genyun Sun ${ }^{\mathrm{c}, \mathrm{g}, *}$, Huimin \\ Zhao $^{\mathrm{d}, *}$, Zhijing Yange,*, Zheng Wang ${ }^{\mathrm{f}, *}$, Stephen Marshall ${ }^{\mathrm{a}}$ \\ ${ }^{a}$ Centre for Signal and Image Processing, University of Strathclyde, Glasgow, U.K. \\ ${ }^{b}$ Electro-Optics, Image 85 Signal Processing, Centre for Electronics Warfare, Cranfield \\ University, Swindon, U.K \\ ${ }^{c}$ School of Geoscience, China University of Petroleum, Qingdao, China \\ ${ }^{d}$ School of Computer Sciences, Guangdong Polytechnic Normal University, Guangzhou, \\ China \\ eSchool of Electronic Information, Guangdong University of Technology, Guangzhou, China \\ ${ }^{f}$ School of Computer Software, Tianjin University, Tianjin, China \\ ${ }^{g}$ Laboratory for Marine Mineral Resources, Qingdao National Laboratory for Marine \\ Science and Technology, Qingdao, China
}

\begin{abstract}
Band selection plays an important role in hyperspectral data analysis as it can improve the performance of data analysis without losing information about the constitution of the underlying data. We propose a MIMR-DGSA algorithm for band selection by following the Maximum-Information-Minimum-Redundancy (MIMR) criterion that maximises the information carried by individual features of a subset and minimises redundant information between them. Subsets are generated with a modified Discrete Gravitational Search Algorithm (DGSA) where we definine a neighbourhood concept for feature subsets. A fast algorithm for pairwise mutual information calculation that incorporates variable bandwidths of hyperspectral bands called VarBWFastMI is also developed. Classification results on three hyperspectral remote sensing datasets show that the proposed MIMR-DGSA performs similar to the original MIMR with Clonal Selection Algorithm (CSA) but is computationally more efficient and easier to

\footnotetext{
* Corresponding authors

Email addresses: genyunsun@163.com (Genyun Sun), zhaohuimin@gpnu.edu.cn (Huimin Zhao), yzhj@gdut.edu.cn (Zhijing Yang), wzheng@tju.edu.cn (Zheng Wang)
} 
handle as it has fewer parameters for tuning.

Keywords: Band selection, discrete optimisation, entropy, evolutionary computation, feature selection, gravitational search algorithm, hyperspectral imaging, Maximum-Information-Minimum-Redundancy, mutual information.

\section{Introduction}

Hyperspectral data is inherently complex because it contains data in both the spatial and spectral domain in a three dimensional data structure. Most HSI cameras record up to several hundred wavebands. Depending on the final ap-

5 plication, much of the recorded data may be unnecessary to retrieve the desired information. In fact, too much information might even have a detrimental effect on data analysis due to the well-known Hughes Phenomenon. Reducing the number of features also results in less storage requirements and computational complexity and minimises the risk of over-fitting.

Traditionally, there are two forms of dimensionality reduction, Feature extraction and feature selection. Feature extraction characterises the raw data and generates new features from the available ones by linear combinations of the same or projecting them onto a lower dimensional subspace. Recent techniques include singular spectrum analysis [1], sparse representation [2,3] or the 15 use of stacked autoencoders [4]. In contrast, feature selection defines the process of selecting a subset of all available features and thereby maintaining the original integrity of the data. The selected subset provides insight into the intrinsic processes that generate the data [5].

In Hyperspectral Imaging (HSI), adjacent bands are typically highly correlated [6] and can safely be removed without significant information loss. Equally, not all recorded wavelengths are meaningful for the individual application and are therefore not essential for the predictive power of the system. In [7], an overview of common state-of-the-art supervised band selection algorithms is given. These incorporate different measures such as the correlation coeffi25 cient, statistical measures like the Chi-Square distribution and most notably 
the Minimal-Redundancy-Max-Relevance (mRMR) criterion that evaluates features by their individual ability to explain class variables while minimising redundancies based on mutual information. In [8], mRMR has been extended and combined with a forward greedy search. Recent supervised techniques also 30 include new concepts such as High Dimensional Model Representation [9]. As ground truth data is rarely available for hyperspectral remote sensing data, unsupervised techniques however provide a more generic approach and are of greater interest for practical applications. Some algorithms have been reviewed in [10], where the hyperspectral bands are ranked by measures such as the Shannon entropy or spectral derivatives. Other approaches include band clustering using various similarity measures and selecting representatives $[11,12]$. Popular similarity measures include information theoretical measures [13] or the correlation coefficient [14]. More sophisticated algorithms try to evaluate a complete band subset rather than individually ranking the features. Generating these subsets is however known to be an NP-hard problem [15]. Therefore, typically Evolutionary Algorithms (EA) are employed to solve such problems. In feature selection, popular EA techniques include Particle Swarm Optimisation (PSO) $[16,17]$ and Firefly Algorithm (FA) [18, 19]. Both algorithms are population based algorithms, where each solution is represented by a particle or firefly re45 spectively. In PSO, particles move within the solution space based on their own best position and a global optimal position. FA extends this concept and introduces interaction between all solutions to allow better optimisation. These concepts all define solutions as a binary mask determining the presence or absence in the selected feature subset and therefore implicitly solve the question of the optimal number of selected bands. In [20] and [21], optimised versions of PSO and FA for hyperspectal band selection with a fixed number of bands are proposed. This has the advantage of giving the user power over the size of the band subset. The solutions are encoded as indices of the selected bands. [6] use a different approach named Clonal Selection Algorithm (CSA), where solutions are represented by immune system antigens that clone and mutate based on the quality of the solution. CSA is used to optimise the Maximum-Information- 
Minimum-Redundancy (MIMR) criterion. Based on entropy and mutual information, the criterion tries to identify subsets with features that individually carry maximum information (entropy) while minimising the redundancy (mutual information) between them. As demonstrated in [6], MIMR-CSA poses a state-of-the-art unsupervised hyperspectral band selection algorithm that outperforms most existing algorithms.

A common problem all of the above mentioned EAs face is the number of control parameters [22] based on the objective function and the constitution of ${ }_{65}$ the dataset. CSA in particular requires six parameters with control parameters for mutation, cloning and selection that need individual tuning. In addition, CSA has a relatively high number of evaluations because the cloning can lead to a very large amount of potential solutions. In this paper, we analyse the suitability of other EAs to solve the band subset generation problem. In addi70 tion, we develope a modified Discrete Gravitational Search Algorithm (DGSA) based on [23] that addresses the issue of the number of parameters as well as the computational cost.

Many of the aforementioned evaluation criteria depend on the use of information theoretic measures. A pre-calculation of the entropy and mutual 75 information based on kernel density estimation is suggested in [6] to evaluate the MIMR criterion in reasonable time. The naive approach of calculating the pairwise mutual information between all bands quickly becomes very computationally expensive especially for hyperspectral datasets that comprise a large amount of data and can last up to several days or weeks, according to our experiments. In [24], a fast algorithm for the pairwise calculation of mutual information of gene regulatory networks data is proposed. On this basis, we propose a Variable kernel BandWidth Fast pairwise Mutual Information (VarBWFastMI) estimation algorithm that accounts for strongly varying distributions of hyperspectral bands within a dataset and calculates the pairwise mutual in-

85 formation of the bands efficiently. Based on VarBWFastMI, the discussed EAs are evaluated on three standard remote sensing HSI datasets and results are analysed with respect to performance, computational cost and reproducibility. 
The two main contributions of this paper can be highlighted as follows: 1) A comprehensive analysis of the calculation of information theoretic measures 90 in hyperspectral data is provided resulting in the VarBWFastMI algorithm. 2) A discrete neighbourhood concept for feature subsets is developed that results in MIMR-DGSA feature selection which is a robust, computationally faster and a less cumbersome algorithm with fewer parameters than similar algorithms.

The rest of the paper is structured as follows: Section 2 introduces the 95 basics of the MIMR criterion. Section 3 establishes details on the proposed VarBWFastMI and MIMR-DGSA algorithms. Section 4 defines some experiments where the algorithm is evaluated and compared with other state-of-theart algorithms. Finally, Section 5 concludes the paper and gives an outlook on possible future work.

\section{2. The MIMR criterion}

The MIMR criterion for band selection is based on information theory, whose fundamental measure, the Shannon entropy $H(X)$ of a random variable $X$ is defined by:

$$
H(X)=-\int_{X} p(x) \log p(x) d x
$$

where $p(x)$ denotes the Probability Density Function (PDF) of $X$.

The information shared by two random variables $X_{1}$ and $X_{2}$ can be measured by the mutual information $I\left(X_{1} ; X_{2}\right)$, which is defined as:

$$
I\left(X_{1} ; X_{2}\right)=\int_{X_{1}} \int_{X_{2}} p\left(x_{1}, x_{2}\right) \log \frac{p\left(x_{1}, x_{2}\right)}{p\left(x_{1}\right) p\left(x_{2}\right)} d x_{1} d x_{2}
$$

where $p\left(x_{1}, x_{2}\right)$ is the joint PDF of random variables $X_{1}$ and $X_{2}$.

To avoid quantisation errors from histogram PDF estimations, a popular method is Parzen window estimation [25]. Given a set of $n$ observations ${ }_{110} x_{1}, x_{2}, \ldots, x_{n}$ of a random variable $X$, its PDF $p(x)$ at point $x$ can be approximated by:

$$
\hat{p}(x)=\frac{1}{n h} \sum_{i=1}^{n} K\left(\frac{x-x_{i}}{h}\right)
$$


where $K($.$) denotes the kernel function or Parzen window and is assumed to be$ a symmetric PDF. $h$ represents the kernel width or bandwidth which controls the smoothness of the resulting density estimate. The choice of $h$ is crucial to the quality of the estimate [26].

The most common kernel function is the Gaussian kernel. The PDF of a univariate random variable with Gaussian kernel can be estimated from $n$ datapoints by:

$$
\hat{p}(x)=\frac{1}{n} \sum_{i=1}^{n} \frac{1}{\sqrt{2 \pi h^{2}}} \exp \left(-\frac{\left(x-x_{i}\right)^{2}}{2 h^{2}}\right)
$$

And for a bivariate distribution:

$$
\hat{p}(x, y)=\frac{1}{n} \sum_{i=1}^{n} \frac{1}{\sqrt{2 \pi h^{2}}} \exp \left(-\frac{\left(x-x_{i}\right)^{2}+\left(y-y_{i}\right)^{2}}{2 h^{2}}\right)
$$

Let $X_{i}$ denote the $i$ th subset of $s$ features and $X_{i_{m}}$ denote the $m$ th feature of that subset with $1 \leq m \leq s$, the MIMR criterion can the be defined by:

$$
\max \left(\sum_{m=1}^{s} H\left(X_{i_{m}}\right)-\frac{2}{s-1} \sum_{1 \leq m_{1}<m_{2} \leq s} I\left(X_{i_{m_{1}}} ; X_{i_{m_{2}}}\right)\right)
$$

As an independent criterion for unsupervised subset evaluation, MIMR maximises the sum of the entropies $H($.$) of the features and minimises the sum of$ the pairwise mutual information $I(. ;$.$) between all features in the subset. The$ higher the entropy of a feature, the higher its information. Equally, the lower the mutual information between two features, the lower the shared information, i.e. the redundancy between them.

\section{The Proposed Algorithm}

A flowchart of the proposed algorithm is outlined in Fig. 1, which has 130 two main steps. The fast calculation of entropy and mutual information with VarBWFastMI and MIMR-DGSA algorithm for band selection. Relevant details are presented in the following Sections. 


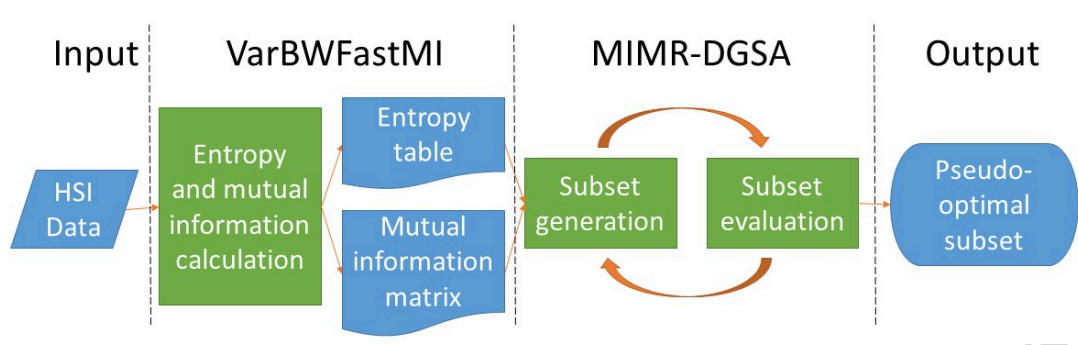

Figure 1: Procedure of the entire band selection algorithm

\subsection{VarBWFastMI}

The estimation of the PDFs of hyperspectral bands to calculate the entropy and mutual information pose some problems in practice that need to be addressed. Depending on the composition of the HSI image, each wavelength can potentially vary very strongly in terms of grey value distribution. Fig. 2 shows the histogram of two different wavelengths of the Indian Pines dataset. It is quite obvious that the two bands contain very different distributions of grey values and require therefore different bandwidths for the kernel density estimation. In this paper, a bandwidth estimation algorithm for Gaussian kernels

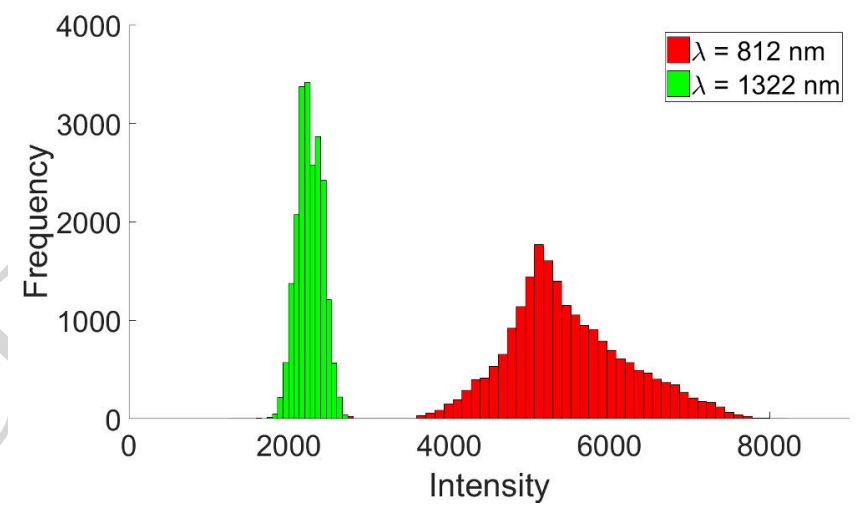

Figure 2: Histogram of wavelengths 812 and 1322nm of Indian Pines dataset. Both wavelength show very different PDFs.

based on the principle of the Mean Integrated Squared Error (MISE) is used. Gaussian kernels are most commonly used and details about the bandwidth es- 
timation can be found in [27]. The bandwidth estimation can deliver a pseudo optimal bandwidth for each hyperspectral band and therefore generate better estimates of the PDF and ultimately the entropy and mutual information. On the basis of the density estimate $\hat{p}_{h}$ with a given bandwidth $h$, an approximation of the entropy $H(X)$ of a band $X$ with $n$ sample points can be directly computed from:

$$
H(X)=\sum_{i=1}^{n} \hat{p}_{h}\left(x_{i}\right) \log \hat{p}_{h}\left(x_{i}\right)
$$

The calculation of the mutual information encounters additional challenges. As seen in Eq. 2, the mutual information requires the joint entropy of the two random variables, which in turn requires a joint density estimate. Using a Gaussian kernel function, the joint density can be estimated by Eq. 5. The computational complexity however rises exponentially and for greater datasets, the cost for the mutual information calculation becomes impractical. In [24], a fast algorithm for calculating the pairwise mutual information between features based on a Gaussian kernel density estimation is introduced for gene regulatory networks. The general idea is to use the fact that the integral of Eq. 2 can be approximated by the sample mean of the respective random variables. The proposed VarBWFastMI makes one major adjustment to that algorithm. Since we estimate a different kernel bandwidth for each hyperspectral band, this needs to be considered for the pairwise mutual information calculation. Given the two bandwidths $h_{x}$ and $h_{y}$ for the two bands $x$ and $y$, Eq. 5 can be rewritten as:

$$
\hat{p}(x, y)=\frac{1}{n} \sum_{i=1}^{n} \frac{1}{\sqrt{2 \pi h_{x} h_{y}}} \times \exp \left(-\frac{1}{2}\left(\frac{\left(x-x_{i}\right)^{2}}{h_{x}^{2}}+\frac{\left(y-y_{i}\right)^{2}}{h_{y}^{2}}\right)\right)
$$

The algorithm in [24] can simply be adapted to estimate the joint densities 165 by Eq. 8 to incorporate the variable bandwidths that are estimated. The implementation of VarBWFastMI is based on the Matlab implementation of the fast pairwise mutual information available at [28]. The code is altered to incorporate variable bandwidths. 


\subsection{DGSA for hyperspectral band selection}

As a heuristic optimisation strategy developed in 2009 [29] that is inspired by Newton's gravitational laws, GSA has gained increasing popularity in recent years in various fields of computation [30, 31]. GSA interprets solutions as objects in an $N$-dimensional space that attract each other according to their mass and gravitational force, where higher masses represent better solutions. The basic concept is visualised in Fig. 3a. In [23], a discrete GSA (DGSA) is developed. Adapted to the problem of hyperspectral band subset generation in particular, DGSA can be defined as follows. Let each band subset $X_{i}=\left(x_{1}, x_{2}, \ldots, x_{N}\right)$ be encoded by agents in an $N$-dimensional space, where $N$ defines the number of desired bands. In each dimension $d$, the possible agent coordinates $x_{d} \in 1,2, \ldots, M$ equal indices of all available bands $F=\left\{f_{1}, f_{2}, \ldots, f_{M}\right\}$. Duplicates are not allowed, i.e. $\forall i, j \in\{1,2, \ldots, N, i \neq j\} \rightarrow x_{i} \neq x_{j}$ which means that no agent can have the same coordinate in more than one dimension. This is to avoid a subset containing the same band more than once. The representation of band subsets by agents in a search space is illustrated in Fig. 3b. The mass $M_{i}$ of

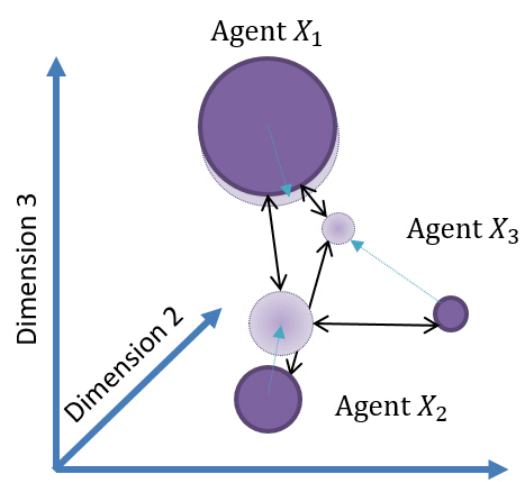

Dimension 1

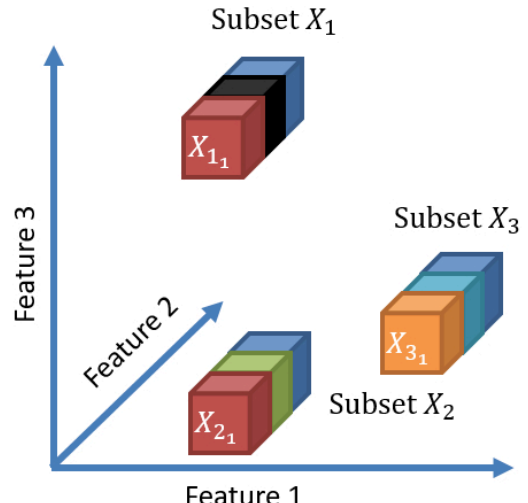

(b)

(a)

Figure 3: DGSA for 3 features. (a) illustrates the agents and their interactions in the three dimensional space and (b) the representation of band subsets through agents. 
to $j$ as well as the normalised distance $N R_{i j}$ are calculated according to [23]. The concept of distance between two band subsets is however redefined here and inspired by the Manhattan distance. The distance between agents $i$ and $j$ can be interpreted as the number of edges that need to be passed along an undirected graph that connects all agents to reach $j$ from $i$. One step can in this context be interpreted as moving agent $i$ one edge along an $N$-dimensional grid towards agent $j$. Note that the movement is ambiguous as no preference as to which neighbour is selected is given in case of multiple paths between the two agents. Fig. 4 illustrates movement in the Manhattan distance space between two agents for a two-dimensional search space. Two possible paths are

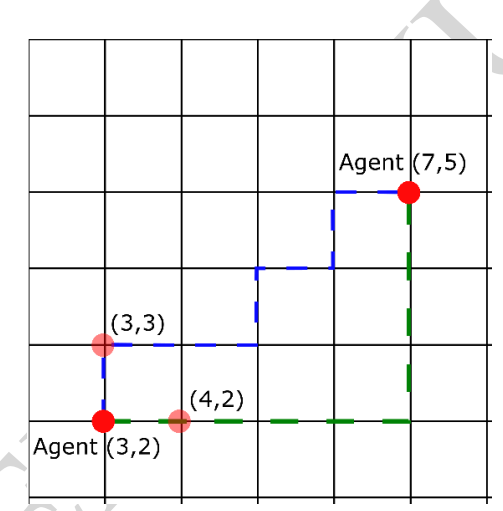

Figure 4: Illustration of movement in Manhattan distance space in a two dimensional space. The agent at coordinates $(3,2)$ is moving towards the agent at $(7,5)$ and has two options at each step. This creates a number of possible shortest paths which are all equally likely.

195

indicated, but the number of paths is not limited to two. As a consequence, each of the possible paths is equally likely. One constraint here is that no band can be selected twice, which means that the number of possible neighbours is reduced. Paths are chosen randomly to introduce a stochastic component and prevent premature convergence.

The resulting MIMR-DGSA algorithm is summarised in Algorithm 1. The initial population $P$ of agents is generated by a randmonised greedy initialisation where $n_{c}$ random bands out of all are selected as candidates and the one that 





produces the highest fitness is chosen and added to a set until the set reaches the desired size $n_{b}$. In the main loop, both $K$ and the gravitational constant $G$ are reduced by a linear reduction function. $K$ should equal 1 in the last iteration. As suggested in [23], the Kbest set contains the globally best agents out of all iterations instead of the local best agents of the current iteration. Mass and acceleration of each agent are calculated according to [23]. In the movement stage, it needs to be specified in which order the $K$ best agents exert their force onto other agents. As stated in [23], later movements have a more significant impact on the quality of the solution, which is why the priority is calculated by the inverse mass. At the end of each iteration, a local search is performed for each agent. This is based on the Hill climbing algorithm. The worst performing band of the current subset is replaced by the best performing band of all remaining bands, defined by the maximum entropy. The search terminates when no neighbour can improve the fitness of the subset. The algorithm terminates after iter $_{\max }$ iterations and the current best solution poses the pseudo-optimal subset of selected bands.

\section{Experimental Results}

For performance assessment, the proposed MIMR-DGSA algorithm was tested on three different hyperspectral remote sensing datasets. Details of the datasets and comprehensive results are discussed in this section as follows.

\subsection{Datasets and experimental setup}

The three hyperspectral datasets include the Indian Pines, the Salinas and the Pavia University. Matlab files for the used datasets can be found in [32]. The Indian Pines dataset was collected by the Airborne Visible/Infrared Imaging Spectrometer (AVIRIS)[33] in 1992 and is a subregion of an image covering the Indian Pines test site in North-western Indiana. It consists of $145 \times 145$ pixels and 224 spectral reflectance bands ranging from 400nm - 2500nm. It contains two thirds agriculture and one third forest and other vegetation. The ground truth is divided in 16 classes as shown in Table 1. 
Table 1: Indian Pines dataset with ground truth classes and their description as well as number of samples available in each class

\begin{tabular}{ccccc}
\hline Class \# & Name & \# Samples & Training & Testing \\
\hline \hline 1 & Alfalfa & 46 & 9 & 37 \\
2 & Corn-notill & 1428 & 285 & 1143 \\
3 & Corn-mintill & 830 & 166 & 664 \\
4 & Corn & 237 & 47 & 190 \\
5 & Grass-pasture & 483 & 96 & 387 \\
6 & Grass-trees & 730 & 146 & 584 \\
7 & Grass-pasture-mowed & 28 & 5 & 23 \\
8 & Hay-windrowed & 478 & 97 & 381 \\
9 & Oats & 20 & 4 & 16 \\
10 & Soybean-notill & 972 & 194 & 778 \\
11 & Soybean-mintill & 2455 & 491 & 1964 \\
12 & Soybean-clean & 593 & 118 & 475 \\
13 & Wheat & 205 & 41 & 164 \\
14 & Woods & 1265 & 320 & 945 \\
15 & Buildings-Grass-Trees-Drives & 386 & 77 & 309 \\
16 & Stone-Steel-Towers & 93 & 18 & 75 \\
\hline
\end{tabular}

The Salinas dataset was also collected by the AVIRIS sensor over the Salinas Valley, California. The dataset comprises $512 \times 217$ pixels and again 224 bands and has therefore a significantly higher data amount than the Indian Pines scene. The scene depicts vegetables, bare soils, and vineyard fields. The ground truth also contains 16 classes, depicted in Fig. 2. To reduce noise effects in the data, the water absorption band regions were removed, i.e. bands [104 - 108], [150 - 163] and 220 for both the Salinas and Indian Pines datasets.

The Pavia University dataset was acquired by the Reflective Optics System Imaging Spectrometer (ROSIS)[34] during a flight campaign over Pavia, Northern Italy. It consists of $610 \times 610$ samples and 103 spectral bands covering a range within 430nm - 860nm. As shown in Fig. 3, the ground truth contains 9 classes.

To evaluate the performance of the proposed MIMR-DGSA algorithm, it was 
Table 2: Salinas dataset with ground truth classes and their description as well as number of samples available in each class

\begin{tabular}{ccccc}
\hline Class \# & Name & \# Samples & Training & Testing \\
\hline \hline 1 & Brocoli_green_weeds_1 & 2009 & 401 & 1608 \\
2 & Brocoli_green_weeds_2 & 3726 & 745 & 2981 \\
3 & Fallow & 1976 & 395 & 1581 \\
4 & Fallow_rough_plow & 1394 & 278 & 1116 \\
5 & Fallow_smooth & 2678 & 535 & 2143 \\
6 & Stubble & 3959 & 791 & 3168 \\
7 & Celery & 3579 & 715 & 2864 \\
8 & Grapes_untrained & 11271 & 2254 & 9017 \\
9 & Soil_vinyard_develop & 6203 & 1240 & 4963 \\
10 & Corn_senesced_green_weeds & 3278 & 665 & 2613 \\
11 & Lettuce_romaine_4wk & 1068 & 213 & 855 \\
12 & Lettuce_romaine_5wk & 1927 & 385 & 1542 \\
13 & Lettuce_romaine_6wk & 916 & 183 & 733 \\
14 & Lettuce_romaine_7wk & 1070 & 214 & 856 \\
15 & Vinyard_untrained & 7268 & 1453 & 5815 \\
16 & Vinyard_vertical_trellis & 1807 & 361 & 1446 \\
\hline
\end{tabular}

Table 3: Pavia University dataset with ground truth classes and their description as well as number of samples available in each class

\begin{tabular}{ccccc}
\hline Class \# & Name & \# Samples & Training & Testing \\
\hline \hline 1 & Asphalt & 6631 & 1326 & 5305 \\
2 & Meadows & 18649 & 3729 & 14920 \\
3 & Gravel & 2099 & 419 & 1680 \\
4 & Trees & 3064 & 612 & 2452 \\
5 & Painted metal sheets & 1345 & 269 & 1076 \\
6 & Bare Soil & 5029 & 1005 & 4024 \\
7 & Bitumen & 1330 & 266 & 1064 \\
8 & Self-Blocking Bricks & 3682 & 736 & 2946 \\
9 & Shadows & 947 & 186 & 761 \\
\hline
\end{tabular}

compared with the original CSA version as well as PSO and FA. The individual parameter settings are listed in Table 4. For CSA, the settings are based on [6], 
whereas for PSO and FA, the parameters of the respective literature were used as a basis and were empirically adjusted for optimal results on the data used in this paper. All algorithms are compared with respect to their band selection capabilities and time consumption. The band selection capabilities were assessed with respect to pixel-wise classification. A Support Vector Machine (SVM) with a Radial Basis Function (RBF) kernel whose parameters $\mathrm{C}$ and $\gamma$ were tuned by a grid search, i.e. selecting $20 \%$ of the pixels of each dataset's classes randomly ${ }_{255}$ for training and the remaining $80 \%$ for validation. The test- and validation-set splitting was repeated 10 times for each dataset and 3 runs of each algorithm were performed for each set making it 30 runs per dataset. As a state-of-theart unsupervised feature selection benchmark for the classification performance, Ward's Linkage strategy using Mutual Information (WaLuMI)[35] algorithm was applied. It hierarchically groups the spectral bands by a distance measure based on mutual information and selects a representative of each group as the band subset. WaLuMI was chosen as it performs best among all compared algorithms in [6] and therefore serves as a baseline. To compare the performance of the MIMR criterion, the Fuzzy C-Means clustering method (FCM) has also been applied in combination with DGSA and ultimately, a classification using all bands of each dataset was compared with that of the selected features.

\subsection{Entropy and mutual information}

For each of the three datasets, the lookup tables for the entropy and mutual information were calculated with the proposed VarBWFastMI algorithm. 270 Results are yisualised in Fig. 10 along the class mean spectra of each class in all three datasets. As expected, the Indian Pines and Salinas datasets show a strong structural similarity for both the entropy and mutual information as they are captured with the same sensor and contain similar vegetation scenes. The bands on the edges of the water absorption regions in the Indian Pines and 275 Salinas datasets show a very low entropy and a low mutual information with the rest of the bands. Wavelength numbers 40 - 100 seem to carry the most information as the entropy is the highest in that range. The Pavia University 
Table 4: Parameter configurations for the different algorithms

\begin{tabular}{|c|c|c|c|}
\hline \multicolumn{2}{|l|}{ CSA } & \multicolumn{2}{|l|}{ DGSA } \\
\hline Population size $s$ & 50 & Population size $s$ & 30 \\
\hline Maximum iterations iter $_{\max }$ & 100 & Maximum iterations iter $_{\max }$ & 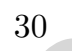 \\
\hline Displaced antibodies $d$ & 5 & Candidates for initialisation $n_{c}$ & \\
\hline Number of clones $n_{c l}$ & 2500 & & \\
\hline Mutation probability $n_{m}$ & 5 & & \\
\hline Selection probability $n_{s}$ & 0.5 & & \\
\hline PSO & & FA & \\
\hline Population size $s$ & 25 & Population size $s$ & 10 \\
\hline Maximum iterations iter $_{\max }$ & 100 & Maximum iterations iter $_{\max }$ & 100 \\
\hline Acceleration coefficient $1 c_{1}$ & 2 & Step size factor $\alpha$ & 0.5 \\
\hline Acceleration coefficient $2 c_{2}$ & 2 & Maximum attraction $\beta_{0}$ & 0.2 \\
\hline Range of inertia weight $w$ & 0.9 & Absorption coefficient $\gamma$ & 1 \\
\hline
\end{tabular}

in contrast contains more man made objects and covers only a portion of the spectral range of the other two datasets and is therefore quite different. It has a remarkably uniform high entropy over almost all bands. In band numbers 70 80, the entropy decreases slightly as the class spectra seem to be less distributed and closer together. The mutual information equally shows a decline as most bands seem to undergo a distinct change specific to their class.

The speed-up achieved by the VarBWFastMI algorithm compared to the naive approach is compared in Tables 5 and 6 . The naive approach entails estimating the univariate and bivariate PDFs of each band and band combinations individually, while for the mutual information matrix, only the upper triangle needs to be calculated and the lower one can be mirrored. The kernel density estimation is done with the Matlab KDE Toolbox available at [36]. It employs kd-trees to faster estimate the kernel density. The VarBWFastMI algorithm was implemented in Matlab as well and both algorithms were run on an Intel Core i5 $\mathrm{CPU}$ at $3.20 \mathrm{GHz}$ with $16 \mathrm{~GB}$ RAM and were performed on the Indian Pines dataset. In Table 5, different numbers of bands out of the 200 available ones 
were selected randomly in 5 runs and the mean runtime was measured. In Table 6 , different numbers of samples were used for the calculation. These numbers stem from taking $1 \%, 5 \%, 10 \%, 25 \%$ and $50 \%$ of the samples of each class. One can see that with an increasing number of bands, the speed-up also increases whereas the speed-up seems to be not directly correlated to the number of samples. As each band has a very different PDF, with very different numbers of samples and distribution over the intensity range, the time consumption cannot be linearly scaled up with an increasing number of samples. A definite speed-up factor cannot be established but it is evident that the proposed VarBWFastMI performs much faster than the naive approach by a large factor. Especially for large datasets such as the Salinas scene dataset, this can significantly reduce the computational cost from several weeks to only days.

Table 5: Time consumption in seconds for the mutual information with varying numbers of bands on the Indian Pines dataset with a fixed number of 1031 samples

\begin{tabular}{lcccccc}
\hline \# Bands & $\mathbf{1 0}$ & $\mathbf{2 0}$ & $\mathbf{5 0}$ & $\mathbf{7 5}$ & $\mathbf{1 0 0}$ & $\mathbf{1 5 0}$ \\
\hline \hline Naive & 53.4 & 303.1 & 1591.7 & 3608 & 6532.1 & 14149 \\
VarBWFastMI & 5.7 & 9.3 & 30.0 & 47.1 & 67.1 & 116.5 \\
\hline Speed-up & 9.3 & 32.6 & 53.1 & 76.6 & 97.5 & 122.0
\end{tabular}

Table 6: Time consumption in seconds for the mutual information with varying numbers of samples for the Indian Pines dataset with all 200 bands

\begin{tabular}{lccccc}
\hline \# Samples & $\mathbf{1 1 0}$ & $\mathbf{5 2 0}$ & $\mathbf{1 0 3 1}$ & $\mathbf{2 5 6 9}$ & $\mathbf{5 1 2 8}$ \\
\hline \hline Naive & 33.1 & 379.4 & 1318.1 & 7249.9 & 25188 \\
VarBWFastMI & 0.6 & 2.3 & 7.9 & 43.7 & 169.6 \\
\hline Speed-up & 55.1 & 165.0 & 166.8 & 165.9 & 148.5
\end{tabular}

Values obtained by the naive approach and VarBWFastMI were compared and differences are at a magnitude of around $10 \times 10^{-5}$. i.e. both approaches yield almost identical results. 


\subsection{DGSA Parameter analysis}

All parameters were plotted against the evaluation of the MIMR fitness function on the mutual information and entropy values for the Indian Pines dataset.

The population size $s$ and number of iterations iter $_{\max }$ are the two parameters

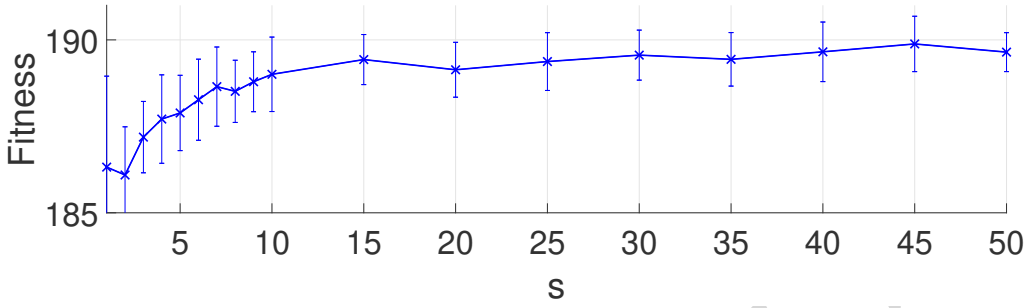

(a)

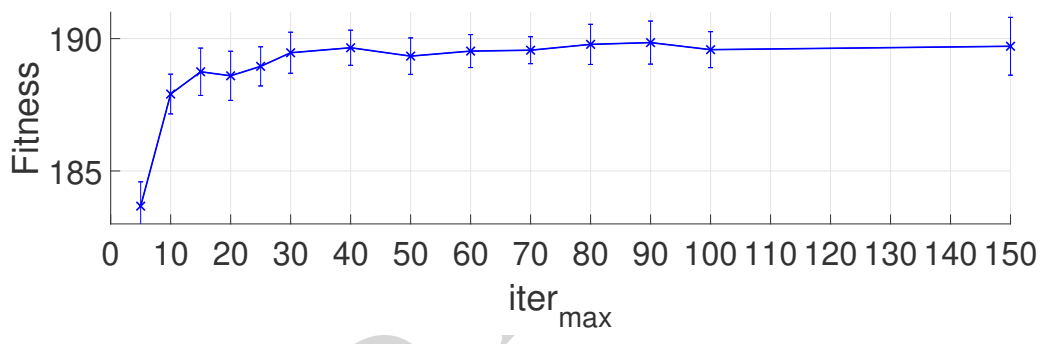

(b)

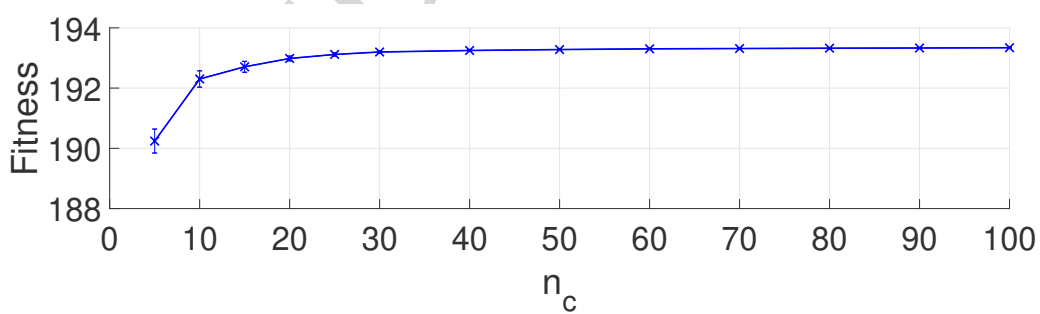

(c)

Figure 5: Sensitivity of DGSA parameters (a) population size (b) number of iterations and (c) number of candidates to the optimisation performance on the Indian Pines Dataset.

influencing the performance of DGSA. Both have been analysed in Fig. 5a and b for a fixed number of 30 bands on the Indian Pines dataset. To magnify the impact of both parameters, DGSA was performed with random initialisation. As 
expected, both parameters increase the performance by increasing their values. They are chosen to be as minimal as possible to achieve maximum optimisation capacity with minimal computational effort. Based on these results, $s$ and iter $\max$ are both set to 30 in future experiments for our MIMR-DGSA method.

The number of candidates $n_{c}$ for the initialisation was analysed in Fig. 5c. By increasing this number, the chances of picking a good solution increase as well. However, to guarantee a good exploration of the search space, a trade-off between subset fitness and population diversity is mandatory. Therefore, we suggest to set $n_{c}$ to 10 .

\subsection{Runtime analysis}

As stated in [20], MIMR-PSO has a computational complextity of $\mathcal{O}(i \times s \times$ $n_{b}^{2}$ ) which is linearly correlated to the number of iterations $i$, the population size $s$ and quadratically correlated to the number of desired bands $n_{b}$ caused by the MIMR evaluation. Due to the interaction between the fireflies in MIMR-FA, it is quadratically correlated to the size of the population and has a complexity of $\mathcal{O}\left(i \times s^{2} \times n_{b}^{2}\right)$. As stated in [6], MIMR-CSA has a time complexity of $\mathcal{O}\left(i \times n_{c l} \times n_{b}^{2}\right)$, which is linearly dependent on the number of clones $n_{c l}$ and quadratically dependent on the number of selected bands $n_{b}$. MIMR-DGSA also has a quadratic complexity, i.e. $\mathcal{O}\left(i \times s \times K \times n_{b}^{2}\right)$, but the number of MIMR evaluations per iterations is limited to the number of initial agents $s$ and the decreasing number of $K$ best solutions. The initialisation of DGSA has a time complexity of $\mathcal{O}\left(s \times n_{c} \times n_{b}^{2}\right)$, which is linearly correlated to the number of candidates $n_{c}$ and quadratically correlated to $n_{b}$. All algorithms share the dependence on number of iteration and the quadratic runtime of the MIMR evaluation, showing the importance of pre-calculating the entropy and mutual information. The main differences are rooted in the population size and the interaction between the solutions. MIMR-DGSA has an additional initialisation step which can potentially decrease the efficiency. In our, case, the number of candidates for the initialisation is relatively low, which is why this step does not have a big effect on the runtime. 


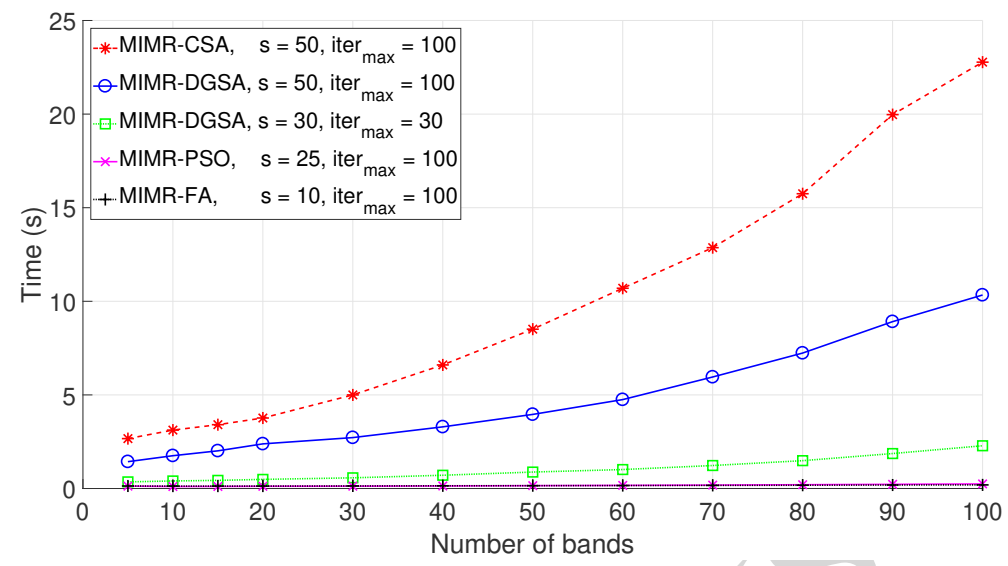

(a)



(b)

Figure 6: Time consumption of (a) MIMR-CSA, MIMR-DGSA with different configurations, MIMR-PSO and MIMR-FA and (b) a closeup of MIMR-DGSA, MIMR-PSO and MIMR-FA with different numbers of selected bands.

To compare the time consumption, all algorithms were performed on the Indian Pines dataset with different numbers of selected bands. For consistency, both CSA and DGSA algorithms were performed with the same population size and are terminated after the same number of iterations. Fig. 5 shows that increasing to number of iterations does not increase the optimisation performance 
significantly. Additionally, MIMR-DGSA was performed with optimised population size and iterations, as established in Section 4.3. The number of candidates for the initialisation of DGSA is set to 10. For PSO and FA, the population size and iterations were set according to [20] and [21] respectively and slightly adjusted based on empirical values and all other parameters are set as specified in Table 4. As seen from the time measurements in Fig. 6, MIMR-DGSA with the same $s$ and iter $_{\max }$ as MIMR-CSA performs about twice as fast. With the parameter settings established in Section 4.3, MIMR-DGSA only requires a fraction of the time of MIMR-CSA, where for 150 features, MIMR-DGSA takes only 5 seconds compared to over 50 seconds for MIMR-CSA. The increased time consumption of CSA is rooted in the relatively high number of clones per antigen, whereas DGSA only has a limited number of agents with a decreasing number of $K$ best agents. PSO and FA both perform very similar and both outperform CSA and DGSA due to their straightforward implementation of the movement strategy. DGSA suffers in this respect due to the elaborate neighbourhood and movement concept.

\subsection{Classification performance}

In this subsection, the classification accuracy using the selected features are compared to evaluate the efficacy of the band selection approaches. MIMR-FA, MIMR-PSO, MIMR-CSA, MIMR-DGSA as well as WaLuMI were performed selecting 30 bands on the Indian Pines and Salinas datasets and 20 bands on the Pavia University dataset. The Overall Accuracy (OA), Average Accuracy (AA) and Kappa coefficient were calculated in every case alongside the individual class accuracies. Results are summarised in Tables 7, 8 and 9 for comparison.

As seen in Table 7, in terms of OA, MIMR-PSO performs best for 30 bands on the Indian Pines dataset, where MIMR-CSA and MIMR-DGSA perform roughly similar. Only FCM-DGSA performs significantly worse. Looking at Fig. 7, One can see that MIMR-DGSA and MIMR-CSA also perform very similar for different numbers of selected bands, outperforming MIMR-FA and WaLuMI, whereas MIMR-PSO performs best selecting 90 or less bands. For the Salinas dataset, 
Table 7: Comparison of classification results selecting 30 bands for different algorithms on the Indian Pines dataset

\begin{tabular}{lllllll}
\hline Class & MIMR- & MIMR- & WaLuMI FCM- & MIMR- & MIMR- \\
& CSA & DGSA & & DGSA & PSO & FA \\
\hline \hline 1 & $72.2 \pm 9.9$ & $75.7 \pm 9.7$ & $71.7 \pm 18$ & $63.8 \pm 13$ & $\mathbf{7 9 . 0} \pm \mathbf{8 . 1}$ & $73.3 \pm 12$ \\
2 & $76.9 \pm 3.0$ & $76.3 \pm 3.6$ & $\mathbf{7 9 . 0} \pm \mathbf{2 . 1}$ & $64.1 \pm 5.4$ & $78.0 \pm 2.9$ & $72.9 \pm 5.9$ \\
3 & $72.5 \pm 3.0$ & $72.0 \pm 3.6$ & $69.7 \pm 3.8$ & $57.2 \pm 5.9$ & $\mathbf{7 3 . 3} \pm \mathbf{3 . 9}$ & $68.6 \pm 7.0$ \\
4 & $67.1 \pm 4.7$ & $68.9 \pm 5.3$ & $\mathbf{6 9 . 1} \pm \mathbf{3 . 2}$ & $51.2 \pm 7.2$ & $67.4 \pm 4.4$ & $64.0 \pm 8.7$ \\
5 & $\mathbf{9 2 . 8} \pm \mathbf{1 . 1}$ & $92.7 \pm 0.9$ & $91.2 \pm 1.1$ & $88.1 \pm 2.3$ & $91.6 \pm 2.2$ & $91.1 \pm 3.0$ \\
6 & $98.0 \pm 1.3$ & $\mathbf{9 8 . 0} \pm \mathbf{0 . 8}$ & $97.0 \pm 1.4$ & $95.1 \pm 1.8$ & $96.5 \pm 1.8$ & $96.2 \pm 1.9$ \\
7 & $88.2 \pm 2.3$ & $86.7 \pm 5.3$ & $\mathbf{8 7 . 3} \pm \mathbf{2 . 0}$ & $60.0 \pm 16$ & $84.8 \pm 9.0$ & $80.0 \pm 10$ \\
8 & $\mathbf{9 8 . 4} \pm \mathbf{0 . 8}$ & $98.0 \pm 1.4$ & $97.7 \pm 1.1$ & $96.6 \pm 2.0$ & $96.9 \pm 1.6$ & $97.0 \pm 1.9$ \\
9 & $63.3 \pm 17$ & $63.8 \pm 19$ & $\mathbf{7 0 . 0} \pm \mathbf{1 8}$ & $22.3 \pm 15$ & $51.0 \pm 24$ & $56.3 \pm 22$ \\
10 & $77.8 \pm 3.2$ & $77.0 \pm 3.3$ & $76.7 \pm 3.1$ & $63.4 \pm 3.5$ & $\mathbf{8 0 . 0} \pm 3.3$ & $71.7 \pm 10$ \\
11 & $\mathbf{8 5 . 3} \pm \mathbf{1 . 3}$ & $84.4 \pm 1.4$ & $83.6 \pm 2.1$ & $78.0 \pm 2.0$ & $85.3 \pm 1.5$ & $82.7 \pm 2.4$ \\
12 & $73.4 \pm 2.9$ & $75.6 \pm 3.8$ & $73.6 \pm 4.9$ & $50.1 \pm 6.9$ & $\mathbf{8 1 . 3} \pm \mathbf{3 . 6}$ & $79.7 \pm 3.6$ \\
13 & $96.2 \pm 1.9$ & $96.3 \pm 2.1$ & $97.2 \pm 2.2$ & $92.0 \pm 4.7$ & $97.3 \pm 1.5$ & $\mathbf{9 8 . 0} \pm 1.2$ \\
14 & $95.8 \pm 0.9$ & $95.7 \pm 0.9$ & $95.1 \pm 1.1$ & $94.4 \pm 1.4$ & $\mathbf{9 5 . 9} \pm \mathbf{1 . 1}$ & $95.5 \pm 1.2$ \\
15 & $59.6 \pm 3.3$ & $60.3 \pm 2.7$ & $56.9 \pm 2.9$ & $47.8 \pm 5.6$ & $\mathbf{6 1 . 8} \pm \mathbf{4 . 7}$ & $59.5 \pm 5.6$ \\
16 & $86.1 \pm 6.8$ & $85.9 \pm 9.0$ & $87.3 \pm 6.8$ & $89.1 \pm 4.3$ & $\mathbf{9 1 . 7} \pm \mathbf{5 . 1}$ & $88.9 \pm 6.1$ \\
\hline OA & $83.6 \pm 0.8$ & $83.4 \pm 0.8$ & $82.9 \pm 0.5$ & $74.4 \pm 2.0$ & $\mathbf{8 4 . 4} \pm \mathbf{1 . 1}$ & $81.5 \pm 2.9$ \\
AA & $81.5 \pm 1.3$ & $81.7 \pm 1.6$ & $81.4 \pm 1.2$ & $69.6 \pm 2.7$ & $\mathbf{8 2 . 0} \pm \mathbf{1 . 8}$ & $79.7 \pm 3.4$ \\
Kappa & $81.3 \pm 1.0$ & $81.0 \pm 0.9$ & $80.5 \pm 0.5$ & $70.6 \pm 2.3$ & $\mathbf{8 2 . 2} \pm \mathbf{1 . 3}$ & $78.8 \pm 3.4$ \\
\hline & & & & & & \\
& & & & & & \\
& & & & & &
\end{tabular}


Table 8: Comparison of classification results selecting 30 bands for different algorithms on the

Salinas dataset

\begin{tabular}{lllllll}
\hline Class & MIMR- & MIMR- & WaLuMI FCM- & MIMR- & MIMR- \\
& CSA & DGSA & & DGSA & PSO & FA \\
\hline \hline 1 & $\mathbf{9 9 . 5} \pm \mathbf{0 . 2}$ & $\mathbf{9 9 . 5} \pm \mathbf{0 . 2}$ & $99.4 \pm 0.2$ & $99.5 \pm 0.4$ & $99.4 \pm 0.4$ & $99.4 \pm 0.4$ \\
2 & $99.8 \pm 0.2$ & $99.8 \pm 0.2$ & $\mathbf{9 9 . 8} \pm \mathbf{0 . 1}$ & $\mathbf{9 9 . 8} \pm \mathbf{0 . 1}$ & $\mathbf{9 9 . 8} \pm \mathbf{0 . 1}$ & $99.7 \pm 0.2$ \\
3 & $98.6 \pm 0.4$ & $98.8 \pm 0.4$ & $\mathbf{9 9 . 6} \pm \mathbf{0 . 2}$ & $99.3 \pm 0.4$ & $99.3 \pm 0.4$ & $99.2 \pm 0.5$ \\
4 & $99.4 \pm 0.2$ & $99.4 \pm 0.3$ & $\mathbf{9 9 . 5} \pm \mathbf{0 . 2}$ & $99.2 \pm 0.4$ & $99.3 \pm 0.4$ & $99.4 \pm 0.4$ \\
5 & $98.2 \pm 0.4$ & $98.4 \pm 0.6$ & $\mathbf{9 9 . 0} \pm \mathbf{0 . 4}$ & $98.9 \pm 0.5$ & $98.9 \pm 0.4$ & $98.8 \pm 0.6$ \\
6 & $99.8 \pm 0.1$ & $99.8 \pm 0.1$ & $\mathbf{9 9 . 9} \pm \mathbf{0 . 1}$ & $\mathbf{9 9 . 9} \pm \mathbf{0 . 1}$ & $99.8 \pm 0.1$ & $99.8 \pm 0.1$ \\
7 & $\mathbf{9 9 . 8} \pm \mathbf{0 . 1}$ & $\mathbf{9 9 . 8} \pm \mathbf{0 . 1}$ & $99.7 \pm 0.1$ & $99.6 \pm 0.2$ & $99.6 \pm 0.2$ & $99.6 \pm 0.2$ \\
8 & $87.5 \pm 0.8$ & $88.3 \pm 0.9$ & $88.6 \pm 0.5$ & $88.8 \pm 0.6$ & $88.8 \pm 0.7$ & $\mathbf{8 9 . 2} \pm \mathbf{0 . 6}$ \\
9 & $98.4 \pm 0.4$ & $99.2 \pm 0.7$ & $\mathbf{9 9 . 8} \pm \mathbf{0 . 2}$ & $99.7 \pm 0.1$ & $\mathbf{9 9 . 8} \pm \mathbf{0 . 1}$ & $\mathbf{9 9 . 8} \pm \mathbf{0 . 1}$ \\
10 & $94.8 \pm 0.6$ & $95.6 \pm 0.8$ & $\mathbf{9 7 . 3} \pm \mathbf{0 . 6}$ & $96.6 \pm 0.9$ & $97.2 \pm 0.6$ & $\mathbf{9 7 . 3} \pm \mathbf{0 . 6}$ \\
11 & $92.7 \pm 1.6$ & $96.1 \pm 2.5$ & $98.8 \pm 0.4$ & $98.3 \pm 0.9$ & $98.4 \pm 1.0$ & $\mathbf{9 9 . 0} \pm \mathbf{0 . 7}$ \\
12 & $99.8 \pm 0.2$ & $99.8 \pm 0.2$ & $99.8 \pm 0.1$ & $\mathbf{9 9 . 9} \pm \mathbf{0 . 1}$ & $99.8 \pm 0.4$ & $\mathbf{9 9 . 9} \pm \mathbf{0 . 1}$ \\
13 & $99.4 \pm 0.3$ & $\mathbf{9 9 . 5} \pm \mathbf{0 . 3}$ & $98.9 \pm 0.6$ & $99.1 \pm 0.6$ & $99.2 \pm 0.5$ & $99.2 \pm 0.5$ \\
14 & $97.7 \pm 1.0$ & $97.9 \pm 0.9$ & $97.1 \pm 1.0$ & $97.4 \pm 1.3$ & $97.4 \pm 1.3$ & $\mathbf{9 8 . 0} \pm \mathbf{1 . 2}$ \\
15 & $71.5 \pm 1.4$ & $74.3 \pm 2.3$ & $73.6 \pm 1.1$ & $75.2 \pm 1.3$ & $74.6 \pm 2.6$ & $\mathbf{7 5 . 8} \pm \mathbf{2 . 2}$ \\
16 & $\mathbf{9 9 . 1} \pm \mathbf{0 . 2}$ & $\mathbf{9 9 . 1} \pm \mathbf{0 . 2}$ & $98.9 \pm 0.2$ & $98.8 \pm 0.3$ & $98.8 \pm 0.3$ & $98.9 \pm 0.3$ \\
\hline OA & $92.6 \pm 0.3$ & $93.4 \pm 0.5$ & $93.6 \pm 0.1$ & $93.8 \pm 0.2$ & $93.8 \pm 0.4$ & $\mathbf{9 4 . 0} \pm \mathbf{0 . 4}$ \\
AA & $96.0 \pm 0.2$ & $96.6 \pm 0.4$ & $96.9 \pm 0.1$ & $96.9 \pm 0.2$ & $96.9 \pm 0.3$ & $\mathbf{9 7 . 1} \pm \mathbf{0 . 3}$ \\
Kappa & $91.8 \pm 0.3$ & $92.6 \pm 0.6$ & $92.9 \pm 0.1$ & $93.1 \pm 0.3$ & $93.0 \pm 0.5$ & $\mathbf{9 3 . 3} \pm \mathbf{0 . 4}$ \\
\hline & & & & & &
\end{tabular}


Table 9: Comparison of classification results selecting 20 bands for different algorithms on the Pavia University dataset

\begin{tabular}{lllllll}
\hline Class & MIMR- & MIMR- & WaLuMI FCM- & MIMR- & MIMR- \\
& CSA & DGSA & & DGSA & PSO & FA \\
\hline \hline 1 & $92.2 \pm 0.6$ & $92.1 \pm 1.0$ & $92.9 \pm 0.4$ & $90.3 \pm 1.2$ & $91.7 \pm 1.4$ & $\mathbf{9 3 . 5} \pm \mathbf{0 . 8}$ \\
2 & $\mathbf{9 7 . 9} \pm \mathbf{0 . 2}$ & $97.7 \pm 0.2$ & $96.9 \pm 0.2$ & $96.5 \pm 0.5$ & $97.0 \pm 0.5$ & $97.1 \pm 0.5$ \\
3 & $75.3 \pm 1.7$ & $74.7 \pm 3.7$ & $76.0 \pm 1.0$ & $66.8 \pm 6.9$ & $74.9 \pm 5.1$ & $\mathbf{7 7 . 4} \pm \mathbf{3 . 3}$ \\
4 & $\mathbf{9 4 . 1} \pm \mathbf{0 . 8}$ & $93.8 \pm 0.9$ & $91.5 \pm 0.7$ & $90.4 \pm 1.1$ & $92.3 \pm 1.2$ & $92.7 \pm 1.4$ \\
5 & $99.3 \pm 0.3$ & $\mathbf{9 9 . 4} \pm \mathbf{0 . 3}$ & $99.3 \pm 0.2$ & $98.5 \pm 0.6$ & $99.1 \pm 0.3$ & $99.2 \pm 0.3$ \\
6 & $\mathbf{8 9 . 6} \pm \mathbf{0 . 6}$ & $88.4 \pm 1.1$ & $81.1 \pm 1.1$ & $64.6 \pm 9.9$ & $77.4 \pm 9.0$ & $83.0 \pm 5.5$ \\
7 & $78.7 \pm 1.8$ & $78.0 \pm 3.4$ & $80.9 \pm 1.7$ & $80.2 \pm 2.1$ & $82.2 \pm 1.9$ & $\mathbf{8 3 . 8} \pm \mathbf{1 . 7}$ \\
8 & $87.4 \pm 0.9$ & $87.6 \pm 1.6$ & $87.9 \pm 0.7$ & $86.8 \pm 1.8$ & $88.7 \pm 1.6$ & $\mathbf{8 9 . 8} \pm \mathbf{1 . 1}$ \\
9 & $99.9 \pm 0.2$ & $\mathbf{9 9 . 9} \pm \mathbf{0 . 1}$ & $99.8 \pm 0.2$ & $99.7 \pm 0.2$ & $99.8 \pm 0.1$ & $99.8 \pm 0.2$ \\
\hline OA & $\mathbf{9 3 . 2} \pm \mathbf{0 . 1}$ & $92.9 \pm 0.5$ & $91.9 \pm 0.1$ & $88.7 \pm 1.8$ & $91.4 \pm 1.7$ & $92.7 \pm 1.1$ \\
AA & $90.5 \pm 0.3$ & $90.2 \pm 0.9$ & $89.6 \pm 0.2$ & $86.0 \pm 2.2$ & $89.2 \pm 1.9$ & $\mathbf{9 0 . 7} \pm \mathbf{1 . 2}$ \\
Kappa & $\mathbf{9 1 . 0} \pm \mathbf{0 . 2}$ & $90.6 \pm 0.7$ & $89.2 \pm 0.1$ & $84.8 \pm 2.5$ & $88.5 \pm 2.3$ & $90.3 \pm 1.5$ \\
\hline
\end{tabular}

Table 10: Mean OA, AA and Kappa Coefficient over the three datasets of the different algorithms.

\section{MIMR- MIMR- WaLuMI FCM- MIMR- MIMR- \\ CSA DGSA \\ DGSA PSO FA}

OA $\quad 89.80 \pm 0.40 \quad \mathbf{8 9 . 9 0} \pm \mathbf{0 . 6 0} \quad 89.47 \pm 0.23 \quad 85.63 \pm 1.33 \quad 89.87 \pm 1.07 \quad 89.40 \pm 1.47$

AA $\quad 89.33 \pm 0.60 \quad \mathbf{8 9 . 5 0} \pm \mathbf{0 . 9 7} \quad 89.30 \pm 0.50 \quad 84.17 \pm 1.70 \quad 89.37 \pm 1.33 \quad 89.17 \pm 1.63$

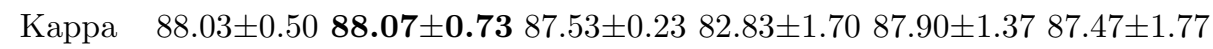


MIMR-FA performs best as seen in Table 8 and Fig. 8. Again MIMR-CSA and MIMR-DGSA show a very similar performance for different numbers of bands but are outperformed by WaLuMI and notably FCM-DGSA for a lower number of bands. For the Pavia University dataset, MIMR in combination with CSA, DGSA, FA and PSO all outperform WaLuMI and FCM-DGSA for any number of bands up to 75, as seen in Fig. 9 where CSA seems to perform best selecting 20 bands. Both MIMR-DGSA and MIMR-CSA again perform very similar as shown in Table 9 and Fig. 9. Another observation that can be made specifically from Tables 7 and 9 is that MIMR-PSO and MIMR-FA yield a larger standard deviation. This might hint at the fact that they have less optimisation qualities for the MIMR criterion and the selected bands are more random, which is examined in Section 4.6. Even though this yields higher mean accuracy, the algorithms are less reliable in individual runs. This leads to the conclusion that FA and PSO can potentially achieve a higher classification accuracy in individual cases, whereas CSA and DGSA perform slightly less but more robust for different datasets. None of the presented algorithms consistently generates optimal performance. To investigate this, we have compared the average measures of the OA, AA and Kappa coefficient over the three datasets, as shown in Table 10. MIMR-DGSA seems to slightly outperform all other algorithms. 400 This hints at the fact that DGSA performs better in terms of generalising over different datasets, but in individual cases, might perform inferior. Hence, even though FA and PSO may produce higher classification accuracy in individual cases, their overall performance for band selection can be compromised due to inferior generalisation capabilities. This is evaluated in detail in the following section.

\subsection{Optimisation performance}

In this section, we are investigating the optimisation performance of the EAs with respect to the MIMR criterion. The objective is to maximise the individual entropies of selected bands and minimise their mutual information. A higher MIMR value indicates a better subset. The actual value is dependent on the 


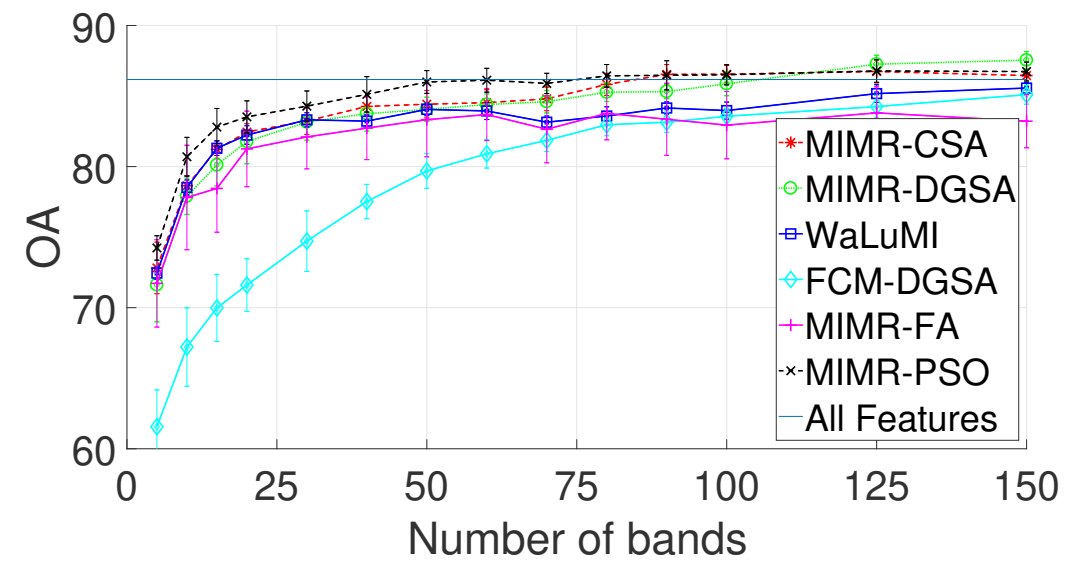

Figure 7: Performance comparison of all band selection algorithms on the Indian Pines dataset

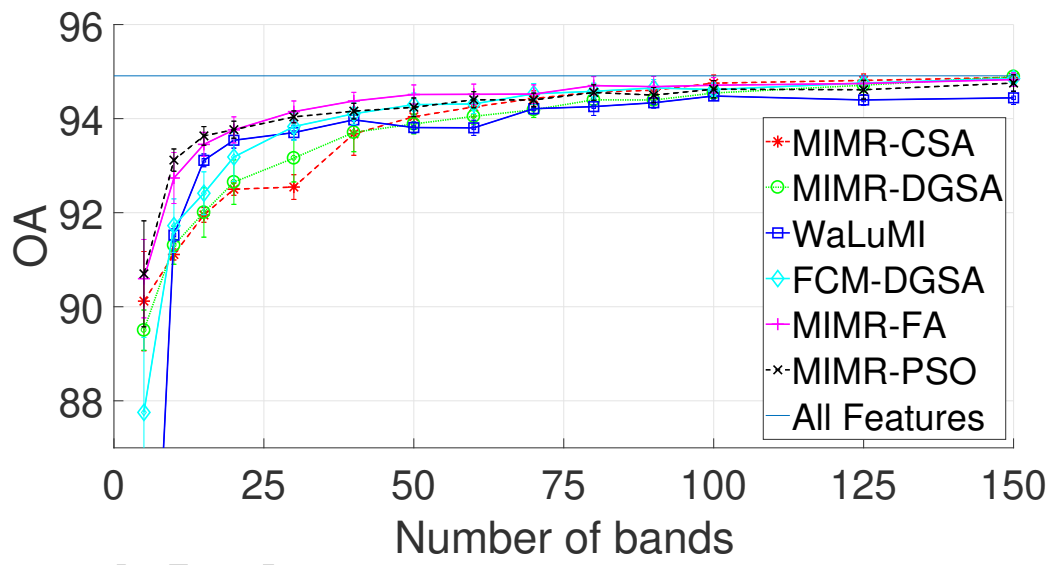

Figure 8: Performance comparison of all band selection algorithms on the Salinas dataset

constitution of each dataset, but algorithms can be compared among each other within one dataset. Table 11 summarises the mean MIMR value achieved after the last iteration of the four EAs of 20 runs. One can see that FA performs worst on all datasets, whereas CSA and DGSA perform very similar. PSO 415 performs better than FA and even becomes even with DGSA for the Pavia University dataset but worse for the other datasets. As seen in Fig. 11, the mutual information and especially the entropy for most bands in the Pavia University 




Figure 9: Performance comparison of all band selection algorithms on the Pavia University dataset

Table 11: Comparison of optimisation performance of the different optimisation algorithms. Values indicate the evaluation of the MIMR criterion of the best solution achieved by each optimisation algorithm.

\begin{tabular}{llllll}
\hline Dataset & \# Bands & FA & PSO & CSA & DGSA \\
\hline \hline Indian Pines & 30 & 175 & 188 & $\mathbf{1 9 3}$ & $\mathbf{1 9 3}$ \\
Salinas & 30 & 159 & 168 & $\mathbf{1 7 3}$ & 172 \\
Pavia University & 20 & 138 & $\mathbf{1 4 1}$ & $\mathbf{1 4 1}$ & $\mathbf{1 4 1}$ \\
\hline
\end{tabular}

dataset are very similar which might suggest that most band combinations yield similar MIMR values. This explains why the optimisation performance of all algorithms is very similar. These findings confirm the above assumption that optimising the MIMR criterion does not necessarily imply an optimal classification accuracy. However, better optimisation of the MIMR criterion results in a more robust accuracy and therefore band selection performance. The fact that FA and PSO yield a better classification accuracy with an inferior optimi-

425 sation capability of the chosen MIMR criterion hence exposes shortcomings of the criterion rather than a superior band selection quality of the optimisation algorithm. For this reason, we consider both CSA and DGSA as more suitable for the proposed task. 


\subsection{Validation of selected bands}

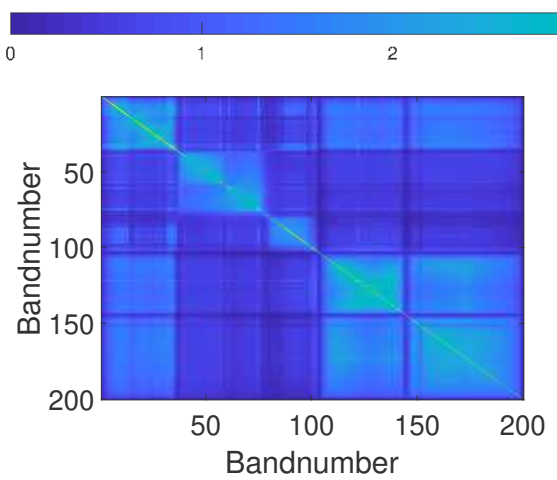

(a)



(c)



(e)

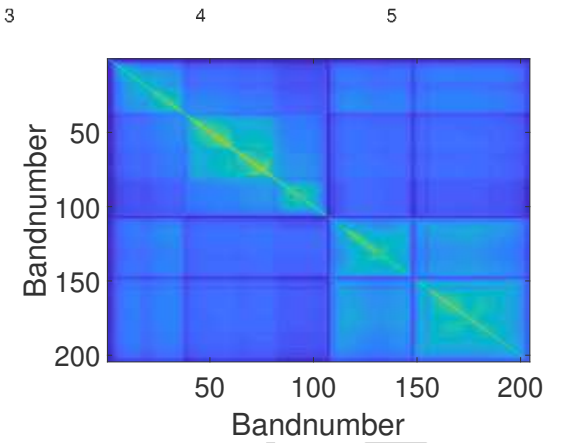

(b)

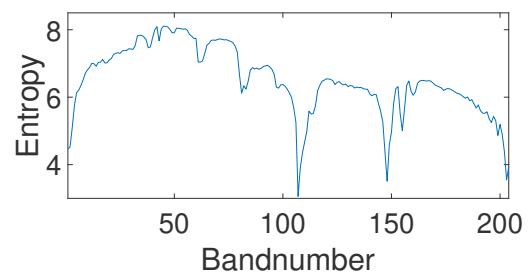

(d)

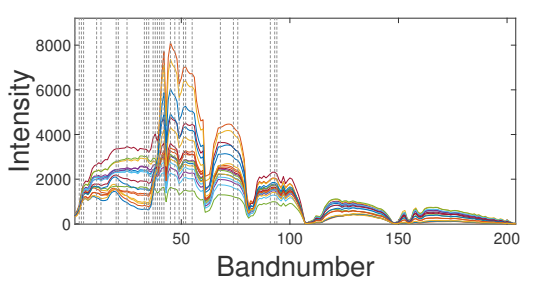

(f)

Figure 10: Comparison of (a) - (b) mutual information, (c) - (d) entropy and (e) - (f) selected bands with DGSA where (a), (c) and (e) refer to the Indian Pines and (b), (d) and (f) to the Salinas dataset. The selected bands could achieve $83.7 \%$ OA for Indian Pines and $92.6 \%$ for Salinas

To verify the selected bands of MIMR-DGSA, the bands of a representative run were plotted on top of the mean spectra of each class in all datasets in Fig. 10 and 11 alongside the mutual information matrices and entropy tables. For the Indian Pines dataset, bands 14 - 16, 20, 23, 26, 29, 31, 34 - 39, 41, 43 - 


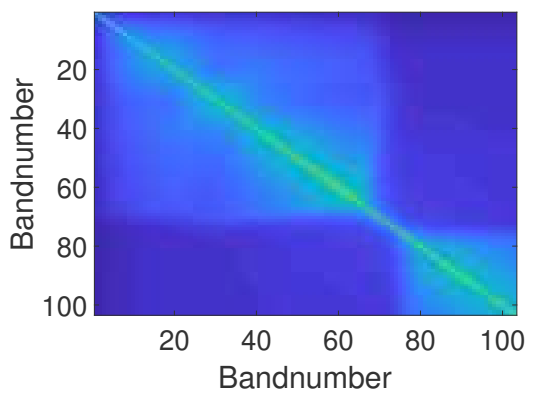

(a)



(b)

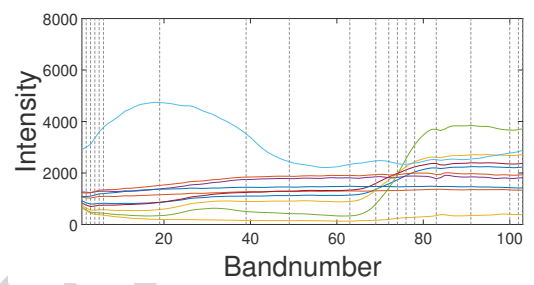

(c)

Figure 11: Comparison of (a) mutual information, (b) entropy and (c) selected bands with DGSA of the Pavia University dataset with an OA of $93.5 \%$.

$45,49,54,62,63,65,68,71,84-86,99$ and 117 were selected. In the Salinas dataset, bands 1,3 - 5, 11, 13, 20, 21, 25, 33 - 35, 37 - 42, 45, 47, 49, 51, 52, 55, 68, 74, 76, 91, 93 and 94 were selected and for the Pavia University dataset, only 20 bands were selected, i.e. 1 - 6, 19, 39, 49, 63, 69, 72, 74, 76, 78, 83, 91, 100, 102 and 103. The bands indexed from 40 to 100 in both the Indian Pines and Salinas scene have the highest entropy and equally a relatively low mutual ${ }_{440}$ information with other regions. Looking at the spectra, this region seems to 
show the biggest differences between the classes, justifying why most bands are selected in this area in both datasets. The bands edging the water absorption regions have a low mutual information but also a very low entropy, which is why they are not selected. The bands higher than 120 have a relatively high entropy but also a quite high mutual information with adjacent bands over a larger region. This is likely the reason why they are hardly ever selected. The mean spectra seem to be very similar in this region as well for most classes. The Pavia University dataset contains the most differences between the classes in the exact wavelength region around number 70 , with a slightly lower entropy but also a much lower mutual information with all other regions. This makes the corresponding region most significant for classification. The edges of all spectra also show some changes in the shapes and are therefore selected. The rest of the bands seem to be evenly distributed over the spectral range.

\section{Conclusion}

In this paper, the MIMR-DGSA algorithm was proposed for hyperspectral band selection. The algorithm is based on the MIMR criterion aiming to maximise the entropy of bands and minimise the mutual information between the bands in a subset. To evaluate the criterion, the entropy and mutual information are pre-calculated. A detailed analysis of the calculation of these measures for hyperspectral bands was presented resulting in the VarBWFastMI algorithm that calculates the pairwise mutual information of hyperspectral datasets in a reasonable amount of time and incorporates variable bandwidths for the density estimation. Additionally, a modified DGSA is developed introducing a neighbourhood and movement concept for feature subsets in a discrete optimisation space that generates potential subsets in a heuristic way and eventually generates a pseudo-optimal solution. Results on three publicly available HSI remote sensing datasets show that the proposed MIMR-DGSA performs very similar to the original MIMR-CSA, on which it is based, but is much faster and easier to use, as it only has three parameters. Parameters for the number of agents and 
iterations are directly correlated with the quality of the optimisation, whereas the number of candidates for the initialisation requires individual tuning based on the underlying dataset.

When comparing both CSA and DGSA with other EAs, we were able to show that FA and PSO are both less suitable to optimise the MIMR criterion.

475 The effect of this is that the achieved classification accuracy is subject to high variance. While PSO and FA can achieve a higher accuracy in individual cases, DGSA was shown to perform superior and be more robust in average over all datasets. This also lead us to conclude that the MIMR criterion itself does not necessarily optimise classification accuracy. In other applications such as regression or object detection, optimising the MIMR criterion might however perform better. Furthermore, PSO and FA require almost as many parameters as CSA and need therefore additional tuning. None of the examined algorithms performs consistently best in terms of accuracy, however, DGSA poses a robust, faster and easier to use alternative to CSA, which both optimise the MIMR criterion best. In combination with VarBWFastMI, we have therefore proposed an efficient state-of-the-art band selection algorithm. In future work, a more robust and faster converging DGSA will be explored, and the conjecture that MIMR performs well for applications other than classification will be examined in detail.

\section{Acknowledgements}

This project is partially funded by Engineering the Future (ETF) Scholarship, Faculty of Engineering, University of Strathclyde; DSTL (R-CLOUD contract DSTLX-1000103251/1000098854); the Fundamental Research Funds for the Central Universities (18CX05030A); Guangdong Provincial Applicationoriented Technical Research and Development Special Fund Project (2016B010127006) and National Nature Science Foundation of China \#U1701266). 


\section{References}

[1] J. Zabalza, J. Ren, J. Zheng, J. Han, H. Zhao, S. Li, S. Marshall, Novel Two-Dimensional Singular Spectrum Analysis for Effective Feature Extraction and Data Classification in Hyperspectral Imaging, IEEE Trans. Geosci. Remote Sens. 53 (8) (2015) 4418-4433.

[2] T. Qiao, Z. Yang, J. Ren, P. Yuen, H. Zhao, G. Sun, S. Marshall, J. A. Benediktsson, Joint bilateral filtering and spectral similarity-based sparse representation: A generic framework for effective feature extraction and data classification in hyperspectral imaging, Pattern Recognit. (2018) 316 -328 .

[3] X. Lu, W. Zhang, X. Li, A Hybrid Sparsity and Distance-Based Discrimination Detector for Hyperspectral Images, IEEE Transactions on Geoscience and Remote Sensing 56 (3) (2018) 1704-1717.

[4] J. Zabalza, J. Ren, J. Zheng, H. Zhao, C. Qing, Z. Yang, P. Du, S. Marshall, Novel segmented stacked autoencoder for effective dimensionality reduction and feature extraction in hyperspectral imaging, Neurocomputing 185 (2016) 1-10.

[5] Y. Saeys, I. Inza, P. Larrañaga, A review of feature selection techniques in bioinformatics, Bioinformatics 23 (19) (2007) 2507-2517.

[6] J. Feng, L. Jiao, F. Liu, T. Sun, X. Zhang, Unsupervised feature selection based on maximum information and minimum redundancy for hyperspectral images, Pattern Recognit. 51 (2016) 295-309.

[7] H. G. Vijouyeh, G. Taskin, A comprehensive evaluation of feature selection algorithms in hyperspectral image classification, in: 2016 IEEE Int. Geoscience and Remote Sensing Symp. (IGARSS), 2016, pp. 489-492. 
[8] Y. Liu, Y. Chen, K. Tan, H. Xie, L. Wang, X. Yan, W. Xie, Z. Xu, Maximum relevance, minimum redundancy band selection based on neighborhood rough set for hyperspectral data classification, Meas. Sci. Technol. 27 (12) (2016) 125501.

[9] G. Taskin, H. Kaya, L. Bruzzone, Feature selection based on high dimensional model representation for hyperspectral images, IEEE Trans. Image Process. 26 (6) (2017) 2918-2928.

[10] P. Bajcsy, P. Groves, Methodology for Hyperspectral Band Selection, Photogramm. Eng. \& Remote Sensing 70 (7) (2004) 793-802.

[11] R. Yang, L. Su, X. Zhao, H. Wan, J. Sun, Representative band selection for hyperspectral image classification, J. Vis. Commun. Image Represent. 48 (2017) 396-403.

${ }_{535}$ [12] M. Zhang, J. Ma, M. Gong, Unsupervised Hyperspectral Band Selection by Fuzzy Clustering With Particle Swarm Optimization, IEEE Geosci. Remote Sens. Lett. 14 (5) (2017) $773-777$.

[13] X. Luo, R. Xue, J. Yin, Information-Assisted Density Peak Index for Hyperspectral Band Selection, IEEE Geosci. Remote Sens. Lett. 14 (10) (2017) $1870-1874$

[14] Q. Du, H. Yang, Similarity-Based Unsupervised Band Selection for Hyperspectral Image Analysis, IEEE Geosci. Remote Sens. Lett. 5 (4) (2008) $564-568$.

[15] E. Amaldi, V. Kann, On the approximability of minimizing nonzero variables or unsatisfied relations in linear systems, Theor. Comput. Sci. 209 (1) (1998) 237-260.

[16] Y. Liu, G. Wang, H. Chen, H. Dong, X. Zhu, S. Wang, An improved particle swarm optimization for feature selection, Journal of Bionic Engineering 8 (2) (2011) 191-200. 
[17] B. Xue, M. Zhang, W. N. Browne, Particle swarm optimization for feature selection in classification: A multi-objective approach, IEEE Transactions on Cybernetics 43 (6) (2013) 1656-1671.

[18] E. Emary, H. M. Zawbaa, K. K. A. Ghany, A. E. Hassanien, B. Parv, Firefly Optimization Algorithm for Feature Selection, in: Proceedings of the 7th Balkan Conference on Informatics Conference - BCI '15, ACM Press, New York, New York, USA, 2015, pp. 1-7.

[19] H. Su, Y. Cai, Q. Du, Firefly-Algorithm-Inspired Framework with Band Selection and Extreme Learning Machine for Hyperspectral Image Classification, IEEE J. Sel. Topics Appl. Earth Obs. Remote Sens. 10 (1) (2017) 309-320.

[20] H. Su, Q. Du, G. Chen, P. Du, Optimized hyperspectral band selection using particle swarm optimization, IEEE Journal of Selected Topics in Applied Earth Observations and Remote Sensing 7 (6) (2014) 2659-2670.

[21] H. Su, B. Yong, Q. Du, Hyperspectral band selection using improved firefly algorithm, IEEE Geoscience and Remote Sensing Letters 13 (1) (2016) 6872.

[22] A. E. Eiben, S. K. Smit, Evolutionary Algorithm Parameters and Methods to Tune Them, in: Autonomous Search, Springer Berlin Heidelberg, Berlin, Heidelberg, 2011, pp. 15-36.

[23] M. B. Dowlatshahi, H. Nezamabadi-Pour, M. Mashinchi, A discrete gravitational search algorithm for solving combinatorial optimization problems, Inf. Sci. 258 (2014) 94-107.

[24] P. Qiu, A. J. Gentles, S. K. Plevritis, Fast calculation of pairwise mutual information for gene regulatory network reconstruction, Comput. Methods Programs Biomed. 94 (2) (2009) 177-180.

[25] E. Parzen, On estimation of a probability density function and mode, Ann. Math. Statist. 33 (3) (1962) 1065-1076. 
[26] N. B. Heidenreich, A. Schindler, S. Sperlich, Bandwidth selection for kernel density estimation: A review of fully automatic selectors, AStA Adv. Statist. Anal. 97 (4) (2013) 403-433.

[27] H. Shimazaki, S. Shinomoto, Kernel bandwidth optimization in spike rate estimation, J. Comput. Neurosci. 29 (1-2) (2010) 171-182.

[28] P. Qiu, Fast calculation of pairwise mutual information based on kernel estimation, Accessed: 02/08/2017. URL http://pengqiu.gatech.edu/software/FastPairMI/index.htm

[29] E. Rashedi, H. Nezamabadi-pour, S. Saryazdi, GSA: A Gravitational Search Algorithm, Information Sciences 179 (13) (2009) 2232-2248.

[30] A. Zhang, G. Sun, J. Ren, X. Li, Z. Wang, X. Jia, A Dynamic Neighborhood Learning-Based Gravitational Search Algorithm, IEEE Trans. Cybern. 48 (1) (2018) 436-447, in press.

[31] G. Sun, P. Ma, J. Ren, A. Zhang, X. Jia, A stability constrained adaptive alpha for gravitational search algorithm, Knowledge-Based Syst. 139 (2018) 200-213, in press.

[32] Hyperspectral remote sensing scenes, Accessed: 14/09/2017.

URL http://www.ehu.eus/ccwintco/index.php?title= Hyperspectral_Remote_Sensing_Scenes

[33] R. O. Green, M. L. Eastwood, C. M. Sarture, T. G. Chrien, M. Aronsson, B. J. Chippendale, J. A. Faust, B. E. Pavri, C. J. Chovit, M. Solis, M. R. Olah, O. Williams, Imaging spectroscopy and the Airborne Visible/Infrared Imaging Spectrometer (AVIRIS), Remote. Sens. Environ. 65 (3) (1998) $227-248$.

[34] S. Holzwarth, A. Müller, M. Habermeyer, R. Richter, A. Hausold, S. Thiemann, P. Strobl, HySens - DAIS 7915 / ROSIS Imaging Spectrometers at DLR, in: 3rd EARSeL Workshop on Imaging Spectroscopy, Herrsching,

605 13-16 May 2003, 2003, p. 12. 
[35] A. Martínez-Usó, F. Pla, J. M. Sotoca, P. García-Sevilla, Clustering-based hyperspectral band selection using information measures, IEEE Trans. Geosci. Remote Sens 45 (12) (2007) 4158-4171.

[36] A. Ihler, Kernel density estimation toolbox for matlab (r13), Accessed: 14/09/2017 (2003).

URL https://www.ics.uci.edu/ ihler/code/kde.html 